\title{
Substitution of amino acid residue V1213 in the helicase domain of the genotype 3 hepatitis $\mathrm{E}$ virus reduces virus replication
}

\author{
Dianjun Cao, Yan-Yan Ni and Xiang-Jin Meng*
}

\begin{abstract}
Background: Genotype 3 hepatitis E virus (HEV) infection is generally associated with mild disease. However, recently eight genotype $3 \mathrm{HEV}$ isolates were identified from patients with severe hepatitis. Importantly, three mutations (S605P, 1978V and V1213A) in these genotype 3 isolates were found to be typical of genotype 4 HEV, which is sometime associated with more severe hepatitis. Therefore in this study we seek to determine if these unique mutations contribute to enhanced virus replication and thus potentially severe disease.

Methods: In the lack of an efficient cell culture system to study the effect of mutations on HEV replication, we developed a genotype $3 \mathrm{HEV}$ replicon with Renilla luciferase (Rluc) as reporter and subsequently used it to construct numerous mutants, including swMu-1 (V1213A), swMu-2 (Q1246H), swMu-3 (V1213A and Q1246H), sWMu-4 (S605P and 1978V), and swMu-5 (V1213A, S605P and 1978V). RNA transcripts from mutant replicons were transfected into Huh7 S10-3 liver cells to measure the effect of mutations on HEV replication efficiency.

Results: The results showed that the V1213A mutant had the highest reduction in HEV replication efficiency than other mutants. The V1213A and S605P + 1978V mutations have a cumulative, if not synergistic, effect on HEV replication. The Q1246H mutant decreased HEV replication compared to the wild-type HEV Rluc replicon but replicated better than the V1213A mutant. The amino acid residue V1213 favors the replication of both genotypes 3 and 4 HEV strains, but not genotype 1 HEV.

Conclusion: The results suggested that the V1213A mutation reduced HEV replication, but is likely not associated with the reported severe hepatitis caused by genotype $3 \mathrm{HEV}$ isolates containing this mutation.
\end{abstract}

Keywords: Hepatitis E virus (HEV), Replication efficiency, Replicon, Pathogenicity

\section{Background}

Hepatitis E virus (HEV) typically causes a self-limiting acute viral hepatitis with large outbreaks reported in individuals from developing countries, and sporadic and cluster cases in individuals from industrialized countries $[1,2]$. More recently, chronic hepatitis E has become a significant clinical problem in immunocompromised individuals such as organ transplant recipients [3]. HEV is a singlestranded, positive-sense RNA virus belonging to the family Hepeviridae [4], which consists of two genera (Orthohepevirus, and Piscihepevirus) and five species. Within the

\footnotetext{
* Correspondence: xjmeng@vt.edu

Department of Biomedical Sciences and Pathobiology, Virginia-Maryland College of Veterinary Medicine, Virginia Polytechnic Institute and State University, 1981 Kraft Drive, Blacksburg, VA 24061-0913, USA
}

species of Orthohepevirus A, there are at least 7 genotypes: genotypes 1 and 2 are restricted to humans whereas genotypes 3 and 4 can cross species barriers infecting humans and several other animal species, and genotype $4 \mathrm{HEV}$ is sometimes reportedly associated with severe acute hepatitis in humans [5]. The genotypes 5 and 6 infect wild boar, and genotype 7 infects camels [4]. The pig is a major animal reservoir for zoonotic transmission of HEV to humans [6]. Indeed, sporadic and cluster cases of acute hepatitis $\mathrm{E}$ are caused predominantly by the zoonotic genotypes 3 and $4 \mathrm{HEV}$ strains $[6,7]$.

The majority of the approximately 20 million HEV infections occurred each year worldwide [8] are asymptomatic, as only about 3 millions of these infections actually resulted in clinical cases of viral hepatitis [9]. The 
mechanisms underlying the induction of liver damage by HEV remains unclear. It has been reported that the genotypes of HEV appear to be associated with diseaseinducing potential [10]. Among the 4 major genotypes of HEV that are known to infect humans, the zoonotic genotypes 3 and 4 HEV isolates are distributed worldwide and have been implicated in sporadic cases of acute hepatitis $\mathrm{E}$ in humans $[1,6,9]$. Interestingly, fulminant or severe acute hepatitis was reported more frequently in humans infected with genotype $4 \mathrm{HEV}$ in Japan [5, 11] and France [12]. Therefore, the virus genotype of the infected patient may potentially influence the severity of liver disease. However, the genetic element(s) in viral genome that are responsible for viral replication and pathogenesis remain unknown.

The genotype $3 \mathrm{HEV}$ is distributed worldwide, and infects humans, pigs, deer, rabbits and other animal species. Recently, increased virulence associated with HEV genotype 3 (JIO strains) infection was reported from patients with severe hepatitis in Japan, although the course of genotype $3 \mathrm{HEV}$ infection is generally asymptomatic [13]. These JIO strains clustered together with 5 swine isolates from Japan [14] and shared an approximately $98 \%$ to 99.8\% nucleotide sequence identity with that of swine HEV, suggesting that these apparently "high virulent strains" of genotype $3 \mathrm{HEV}$ may be of zoonotic origin. There is no reported recombination between the JIO strains of genotype $3 \mathrm{HEV}$ and isolates of genotype $4 \mathrm{HEV}$, but 18 unique amino acid substitutions were identified. Interestingly, three of these mutations (S605P, 1978V, and $\mathrm{V} 1213 \mathrm{~A})$ located in the helicase or protease domain of HEV were found to be typical of genotype 4 viruses which are sometimes associated with more severe hepatitis. Therefore, it is logical to hypothesize that these mutations may be responsible for the increased virulence reported in these genotype $3 \mathrm{HEV}$ strains in humans. Knowing the effects of these mutations on HEV replication will greatly help us understand the mechanism of HEV pathogenesis. Thus, in this study, we determined the effect of the V1213A, S605P, and I978V amino acid residue mutations on the efficiency of HEV replication using the HEV replicon systems, since currently there is a lack of an efficient cell culture system for HEV propagation.

\section{Methods}

\section{Cell cultures, HEV infectious clones, and HEV replicons}

The Huh7-S10-3 liver cell line that supports a limited level of HEV replication and the genotype 1 human HEV infectious clone (Sar55 strain) are gifts from Dr. Sue Emerson (NIH, Bethesda, MD). The Huh7-S10-3 liver cells were cultured in DMEM medium containing $10 \%$ heat-inactivated fetal bovine serum (FBS) at $37{ }^{\circ} \mathrm{C}$ in a $5 \% \mathrm{CO}_{2}$ incubator. The infectious cDNA clones pSHEV3 (genotype 3 swine HEV) and TW9616 (genotype 4 human HEV) were constructed in our laboratory and described previously $[15,16]$. The EGFP replicons of genotype $1 \mathrm{HEV}$ Sar55 and genotype $3 \mathrm{HEV}$ pSHEV3 were constructed previously in our laboratory [17]. The pSK-HEV2-hRluc replicon was described previously [18].

\section{Construction of genotype 3 HEV, and genotype 4 HEV Renilla luciferase (hRluc) replicons}

HEV does not replicate efficiently in cell culture, and therefore in order to study the effect of mutations on HEV replication, an HEV replicon system is needed to more robustly assess the effect of mutations on virus replication efficiency. The infectious cDNA clones of the pSHEV3 and TW6196E were used as the backbone for the construction of genotype 3 and 4 HEV Rluc replicons, respectively. The Rluc replicons were constructed by synthesizing a PCR fusion product containing the 3' ORF1 region of pSHEV3 or TW6196E fused to the Rluc gene and substituting it for the AflII to HpaI region in the pSHEV3 infectious cDNA clone or the AflII to EcoRI region in the TW6196E infectious cDNA clone (Fig. 1a and b). The 3' ORF1 region from pSHEV3 or TW6196E was amplified from the infectious cDNA clones by using the primer set SWHEV4623U and SWHEV5208L or TW4675F and TW5168R, respectively (Table 1). The hRluc gene was amplified from pGL4.70[hRluc] vector (Promega) by using the primer set SWhRluc1U and SWhRluc915L or TWJRhRlucU and TWhRlucL, respectively (Table 1 ). All amplified PCR products were purified with a wizard SV gel and PCR clean-up system (Promega). Subsequently, the pSHEV3- and the TW6196E-related PCR products were mixed, respectively, and the fusion PCR reaction performed. The fusion product was purified by wizard SV gel and PCR clean-up system, digested with AflII-HpaI or AflII-EcoRI accordingly, and ligated into the large fragment of the corresponding infectious cDNA clones from which the AflII-HpaI or AflIIEcoRI region were deleted (Fig $1 \mathrm{~b}$ ). The inserts of both replicons were confirmed by Sanger DNA sequencing.

\section{Construction of mutants of genotype $3 \mathrm{HEV}$ replicon}

The mutants containing the V1213A mutation in ORF1 of HEV genotype 3 GFP replicon (pSHEV3-EGFP) and the A1213V mutation in ORF1 of HEV genotype 1 GFP replicon (pSK-HEV2-EGFP) were constructed with QuikChange $^{\circ}$ II XL Site-Directed Mutagenesis Kit (Stratagene). Using the pSHEV3-Rluc replicon as the backbone, we also constructed 5 mutants with mutations in the ORF1, designated as (1) swMu-1 mutant containing the V1213A mutation; (2) swMu-2 mutant containing the Q1246H mutation; (3) swMu-3 mutant containing the Q1246+V1213A mutations; (4) swMu-4 mutant containing S605P + I978V mutations, and (5) swMu-5 mutant containing S605P + I978V + V1213A 


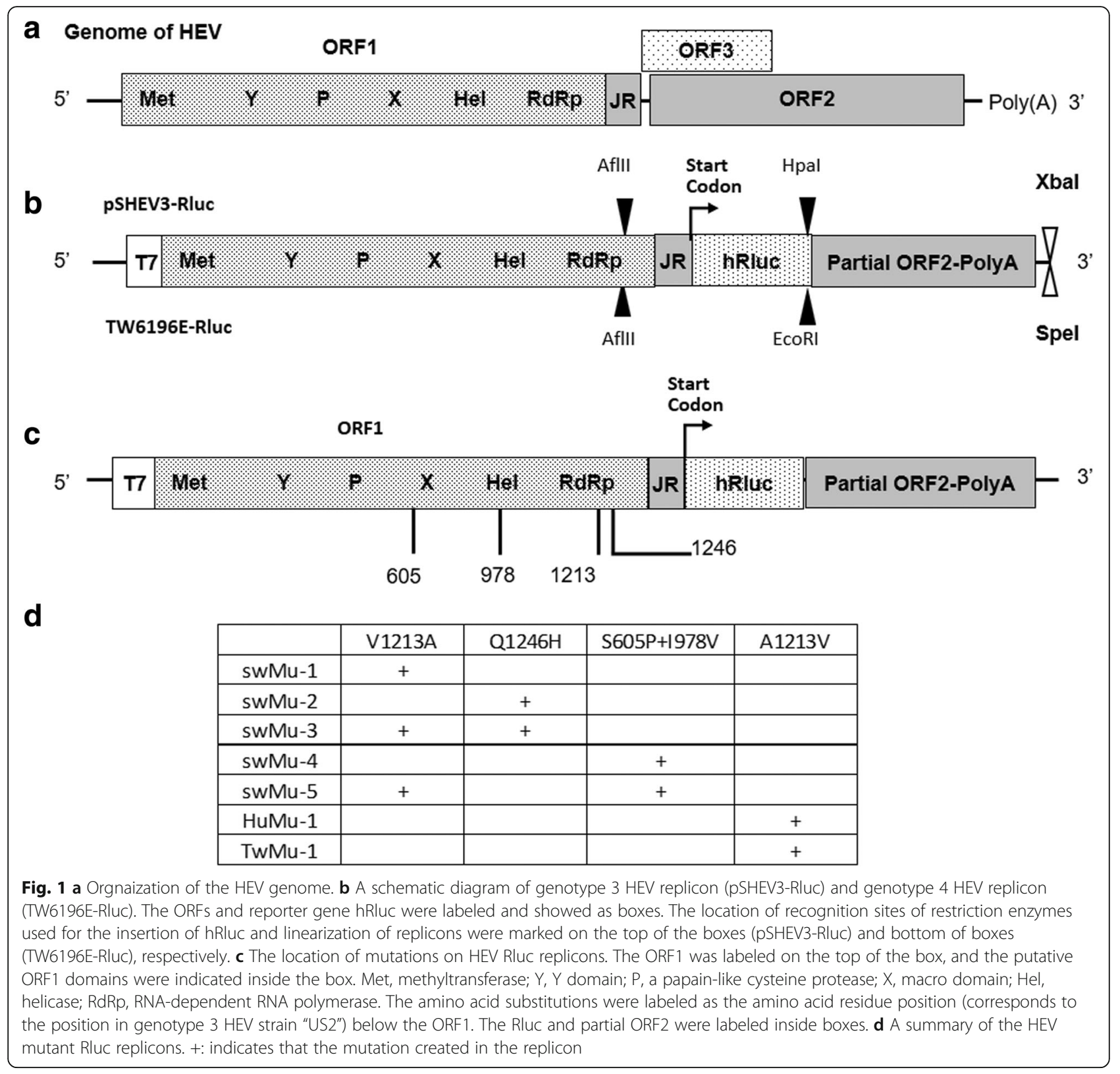

mutations (Fig. 1c and d). The mutants containing single site mutation were generated with QuikChange ${ }^{\odot}$ II XL Site-Directed Mutagenesis Kit (Stratagene) and the mutants containing double or triple mutations were produced with QuikChange ${ }^{\curvearrowleft}$ multi Site-Directed Mutagenesis Kit (Stratagene) according to the manufacturer's instructions. All primers used to introduce the mutations were listed in Table 1.

\section{Construction of mutants of genotype 1 and genotype 4 HEV Rluc replicons}

To assess the impact of amino acid substitution at position 1213 in the ORF1 on virus replication of genotype 1 and genotype $4 \mathrm{HEV}$, we constructed the A1213V mutant replicon in the HEV hRluc replicon of HEV genotype 1 (pSK-HEV2-Rluc) and genotype 4 (TW6196-Rluc), respectively, with QuikChange II XL Site-Directed Mutagenesis Kit (Stratagene). The TW6196-Rluc HEV replicon was constructed in this study, and the primers used for the construction were listed in Table 1.

\section{In vitro RNA transcription and transfection}

The plasmids containing the backbone of pSK-HEV2 (genotype $1 \mathrm{HEV}$ ) were linearized with $\mathrm{BglII}$. The plasmids containing the backbone of pSHEV3 (genotype 3 $\mathrm{HEV}$ ) were digested with $\mathrm{XbaI}$, and the plasmids containing genotype $4 \mathrm{HEV}$ backbone were linearized with SpeI. The purified linearized plasmid DNA was used as 
Table 1 Primers used in the generation of HEV Rluc replicons and their mutants

\begin{tabular}{|c|c|c|c|}
\hline \multicolumn{2}{|l|}{ Primer IDs } & \multirow[t]{2}{*}{ Primer Sequence ${ }^{a}$} & \multirow[t]{2}{*}{ Modifications $^{\mathrm{b}}$} \\
\hline & & & \\
\hline & TW4675F & 5' GCTATGAGTTCCGGGACCTCAA 3' & TW6196-Rluc \\
\hline & TW5168R & 5' GGTGGCATCGCCATGCAA 3' & \\
\hline & TWJRhRlucU & $5^{\prime}$ TTGCATGGCGATGCCACC ATGGCTTCCAAGGTGTACGAC 3' & \\
\hline & TWhRlucL & 5' AGAATTCAGCGCTGCTAGCTATTACTGCTCGTTCTTCAGCACG 3' & \\
\hline & SWhRluc1U & 5' TGCATCGCCCATGGGATCACCATGGCTTCCAAGGTGTACGAC 3' & pSHEV3-Rluc \\
\hline & SWhRluc915L & 5' CGGTTAACATATGCTAGCCTATTACTGCTCGTTCTTCAGCACG 3' & \\
\hline & SWHEV4623U & 5' CGCCGAAGGAGTCTCTTAAGGGT 3' & \\
\hline & SWHEV5208L & 5' GTCGTACACCTTGGAAGCCATGGTGATCCCATGGGCGATGCA 3' & \\
\hline \multicolumn{4}{|l|}{ Mutagenic } \\
\hline & HEV3MvaU & 5' GTGAGGTCGGCATTTCGGATGCAATTGTCAATAACTITITCCTTG 3' & V1213A \\
\hline & HEV3MvaL & 5' CAAGGAAAAAGTTATTGACAATTGCATCCGAAATGCCGACCTCAC 3' & \\
\hline & HEV3MqhU & 5' GATCAGAACCTCGGGACTCTACATGCCTTCCCGCCGTCCTGCCAG 3' & $\mathrm{Q} / \mathrm{H}$ \\
\hline & HEV3MqhL & 5' CTGGCAGGACGGCGGGAAGGCATGTAGAGTCCCGAGGTTCTGATC 3' & \\
\hline & HEV3MspU & 5' GTCTATGGGGGCCGGGCCTCATAGCCTCACTTATG 3' & S605P \\
\hline & HEV3MspL & 5' CATAAGTGAGGCTATGAGGCCCGGCCCCCATAGAC 3' & \\
\hline & HEV3MivU & 5' GCTTGTGCCGGCTGCACTGTTAGTCCTGGGATTG 3' & $1978 \mathrm{~V}$ \\
\hline & HEV3MivL & 5' CAATCCCAGGACTAACAGTGCAGCCGGCACAAGC 3' & \\
\hline & HEV3MvaHU & 5' GTGAGGTCGGCATTTCGGATGCTATTGTCAATAACTTITTCCTTG 3' & V1213A (2nd) \\
\hline & TWMavU & 5' TGAGGTGGGTATCTCTGATGTTATTGTTAACAACTTCTTCC 3' & A1213V TW6196 \\
\hline & TWMavL & 5' GGAAGAAGTTGTTAACAATAACATCAGAGATACCCACCTCA 3' & \\
\hline & HEV2MavU & 5' GCGAGGTGGGCATCTCCGATGTGATCGTTAATAACTITTTCCTTG 3' & A1213V Sar55 \\
\hline & HEV2MavL & 5' CAAGGAAAAAGTTATTAACGATCACATCGGAGATGCCCACCTCGC 3’ & \\
\hline
\end{tabular}

${ }^{\mathrm{a}}$ Sequences of primers

${ }^{\mathrm{b}}$ Rluc inserted into HEV genomes or amino acid mutation introduced by the primer

templates for in vitro transcription of capped RNA with the mMessage mMachine T7 ultra kit (Ambion) as described previously [18].

The capped RNA transcripts from each of the HEV replicons as well as replicons containing mutation(s) were co-transfected together with firefly luciferase RNA into the Huh7-S10-3 liver cells with DMRIE-C reagent (Invitrogen) following the manufacturer's instruction. Huh7-S-10-3 cells that had been transfected with RNA transcripts from HEV replicons or mutants were harvested at 5 days post-transfection. The GFP expression signals were analyzed by flow cytometry as described previously [19]. The luciferase activities were measured with a dual luciferase reporter assay system (Promega), and firefly luciferase signals from the co-transfected firefly luciferase RNA were used to normalize the Renilla luciferase signals $[17,18]$. Relative Luminescence Unit (RLU) is measured at 5 days post-transfection and normalized with the signal from the co-transfected firefly luciferase RNA. Data were collected from an average of eight separate replicate experiments. For the comparison of RLU produced by multiple mutants of HEV Rluc replicons, data were presented as relative luciferase activities to wild-type replicon (the ration of the sample's RLU to that produced by the wild-type replicon), and the RLU for the wild-type Rluc replicons were set as $100 \%$.

\section{Statistical analysis}

Multiple comparisons among the experimental groups were analyzed with one-way analysis of variance (ANOVA) followed with Kruskal-wallis test, and the comparisons between two groups were calculated with two-tailed $t$-test. All data analyses were performed with GraphPad Prism 6 (GraphPad Software, Inc.). The significant difference was defined as $P<0.05$, and the highly significant difference as $p<0.01$.

\section{Results}

The A1213V mutation in genotype $1 \mathrm{HEV}$ and the V1213A mutation in genotype $3 \mathrm{HEV}$ decreased virus replication efficiency

To determine if the mutation at amino acid residue 1213 in genotypes 1 and $3 \mathrm{HEV}$ has any effect on virus replication, we compared the replication levels of HEV 
EGFP replicons containing the A1213V mutation in genotype 1 and V1213A mutation in genotype $3 \mathrm{HEV}$ replicons. A significantly lower number of GFP-positive cells was observed in Huh7 S10-3 liver cells transfected with the genotype 1 pSK-HEV2-EGFP replicon containing the A1213V mutation (Fig 2a). The V1213A mutation in the genotype $3 \mathrm{HEV}$ EGFP replicon showed a slightly but significant lower number of EGFP-positive Huh7 S10-3 cells as well (Fig 2b). The results suggest that the mutation at amino acid residue 1213 reduced the replication level of both genotype 1 and genotype 3 HEV replicons.

However, we found that the EGFP HEV replicon systems are not very sensitive for measuring HEV replication level. For example, the genotype 3 HEV EGFP replicon not only showed lower number of EGFPpositive cells in the transfected cells but also exhibited a lower density of GFP signal in the positive cells. Since both the number of positive cells and the density of positive signals are related to the replication level of HEV replicons, a more sensitive replicon system such as Renilla luciferase replicon is needed to compare the replication level of HEV and their mutants in the absence of an efficient cell culture system for HEV propagation.

\section{HEV luciferase replicon system reflects virus replication more accurately than the HEV EGFP replicon}

By using a Renilla luciferase as the reporter, we developed HEV genotypes 1 and 3 Rluc replicon systems. Subsequently, a V1213A mutant Rluc replicon (swMu-1) was constructed in the HEV genotype 3 Rluc replicon, and a A1213V mutant was constructed in the HEV genotype 1 Rluc replicon. The luciferase activities in Huh7 liver cells transfected with the RNAs from the V1213A and A1213V mutant replicons were measured and analyzed. The results showed that the A1213V mutation in genotype $1 \mathrm{HEV}$ Rluc-replicon reduced HEV replication level similar to what we found with the EGFP replicon system (Fig 2c). The V1213A mutation in HEV genotype 3 Rluc-replicon reduced HEV replication efficiency more significantly than what we observed with the EGFP replicon system (Fig 2d). The data suggested that the HEV luciferase replicon system is more
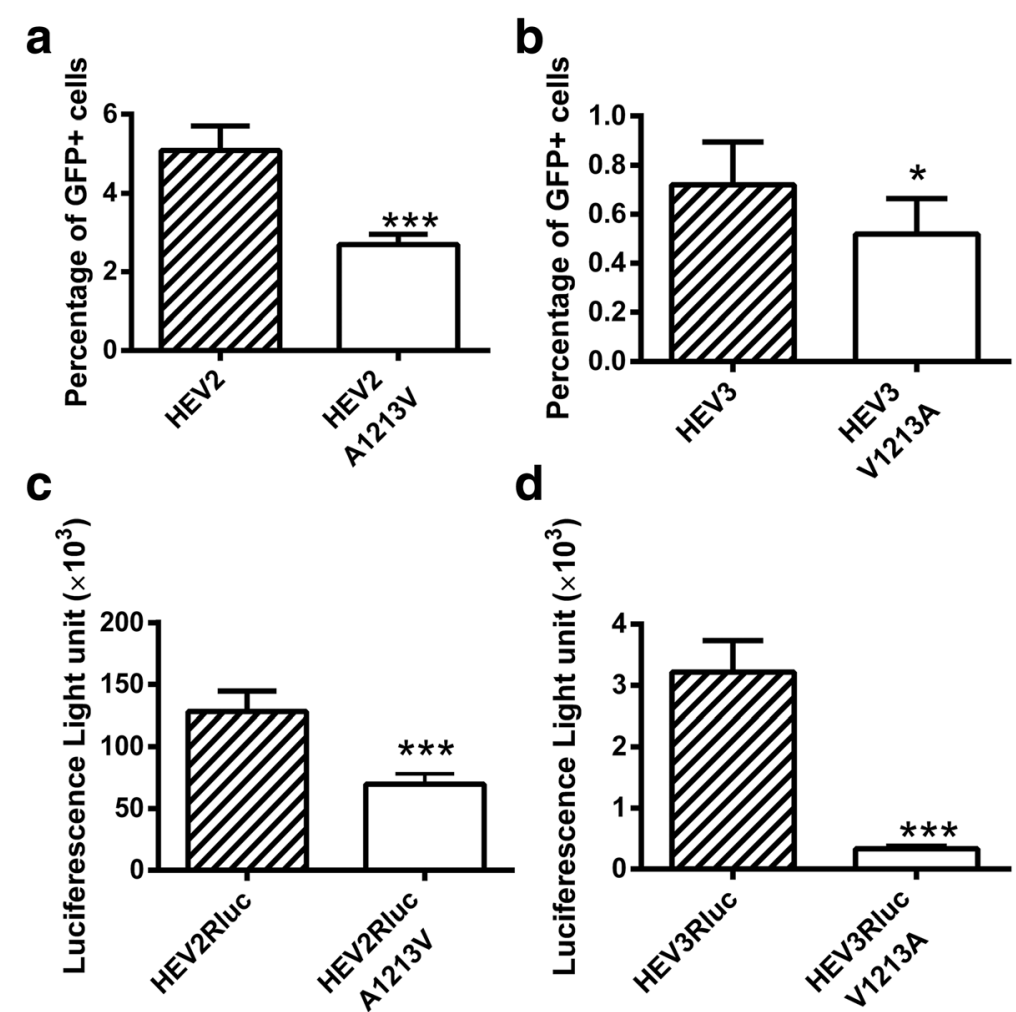

Fig. 2 Effect of mutation at amino acid residue 1213 on genotype 1 and genotype 3 HEV replicons. a A1213V mutation on genotype 1 HEV EGFP replicon. HEV2, pSKHEV2-EGFP replicon; HEV2 A1213V, pSKHEV2-EGFP replicon with mutation A1213V. b V1213A mutation on genotype 3 HEV EGFP replicon. HEV3, pSHEV3-EGFP replicon; HEV3 V1213A, pSHEV3-EGFP replicon with mutation V1213A. c A1213V mutation on genotype 1 HEV luciferase replicon. HEV2Rluc, pSKHEV2-Rluc replicon; HEV2Rluc A1213V, pSKHEV2-Rluc replicon with mutation A1213V. d V1213A mutation on genotype 3 HEV luciferase replicon. HEV3Rluc, pSHEV3-Rluc replicon; HEV3Rluc V1213A, pSHEV3-Rluc replicon with mutation V1213A. The error bars indicate standard deviations (SD). The differences of RLU produced by HEV Rluc mutants and the wild-type Rluc replicon were compared by $t$-test. Asterisks $\left({ }^{*}\right)$ indicate statistical differences compared to the wild-type HEV replicons. ${ }^{*}, P<0.05 ;{ }^{* * *}, P<0.001$ 
sensitive, and reflects HEV replication more accurately than HEV EGFP replicon system.

\section{The S605P and 1978V double mutations in genotype 3} HEV significantly decreased virus replication efficiency When mutations S605P and I978V were introduced into the pSHEV3-Rluc genotype $3 \mathrm{HEV}$ replicon, we found that the dual mutations significantly reduced the replication efficiency of pSHEV3-Rluc HEV replicon, which can be further reduced by introducing the V1213A mutation (Fig 3). The results indicated that the V1213A mutation affected the replication efficiency of genotype $3 \mathrm{HEV}$ more significantly than S605P + I978V mutations. To confirm that the effect of V1213A mutation on HEV replication was caused by amino acid change, not the nucleic acid, we constructed another V1213A mutation with a different condon, GCA, in place of the previous codon GCT. There was no difference on the efficiency of HEV replication when S650P + I978V replicon was combined with different codons of the V1213A mutation, suggesting that the effect of the mutation on HEV replication was caused by the amino acid change.

The Q1246H mutation in genotype $3 \mathrm{HEV}$ reduced virus replication efficiency at a lower level than the V1213A mutation

In addition to the mutations of V1213A, S605P and $\mathrm{I} 978 \mathrm{~V}$, there is also a Q1246H mutation identified in the

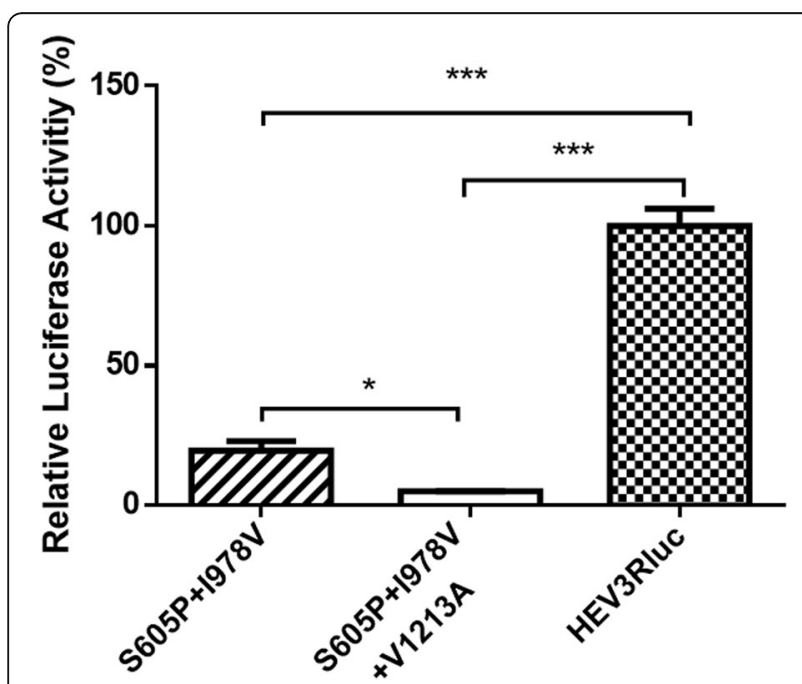

Fig. 3 Relative luciferase activities in Huh7 S10-3 liver cells transfected with RNA of HEV genotype 3 Rluc mutants containing S605P + 1978V, and V1213A mutations. S605P + 1978V: pHEV3-RLuC contains mutations of S605P and 1978V; S605P + 1978V + V1213A: pHEV3-RLuc contains mutations of S605P, 1978V and V1213A. The error bars indicate standard deviations (SD). The differences of relative luciferase activity produced by mutants of HEV Rluc replicons and the wild-type HEV Rluc replicon were compared by one-way analysis of variance (ANOVA) using the Kruskal-Wallis test $(P<0.05)$. Asterisks $\left(^{*}\right)$ indicate statistical differences compared to the parental wild-type HEV replicons, ${ }^{*}, P<0.05 ;{ }^{* *}, P<0.001$ high virulent genotype $3 \mathrm{HEV}$ isolates, but the $\mathrm{H}$ residue is different from the corresponding residue typically found in the genotype 4 isolates [13]. To determine the effect of Q1246H mutation found in the high virulent genotype $3 \mathrm{HEV}$ isolates on the virus replication, a panel of HEV mutants were also constructed in HEV Rlucreplicon to determine if these unique amino acid mutations affect $\mathrm{HEV}$ replication efficiency. In the genotype 3 HEV Rluc replicon system, we found that the V1213A mutation reduced HEV replication efficiency more significantly than the $\mathrm{Q} 1246 \mathrm{H}$ mutation (Fig 4a). The $\mathrm{V} 1213 \mathrm{~A}+\mathrm{Q} 1246 \mathrm{H}$ double mutations had a more significant reduction of $\mathrm{HEV}$ replication level than the Q1246H mutation alone (Fig 4a). The results suggested that the V1213A mutation is more important to the replication of genotype $3 \mathrm{HEV}$ than the Q1246H mutation, and that there was no apparent synergistic effect between V1213A mutation and Q1246H mutation.

\section{The A1213V mutation in genotype 4 HEV significantly increased virus replication efficiency}

Furthermore, to determine if the function of amino acid residue at position 1213 is $\mathrm{HEV}$ genotype-specific, we constructed a A1213V mutant in the genotype $4 \mathrm{HEV}$ Rluc-replicon. Contrary to what we observed with genotype $1 \mathrm{HEV}$, we found that the A1213V mutation significantly increased the replication level of genotype $4 \mathrm{HEV}$ (Fig 4b). The results suggested that the amino acid residue $\mathrm{V}$ at position 1213 actually favors the replication of both zoonotic genotypes 3 and 4, but not genotype 1 .

\section{Discussion}

Genotype $3 \mathrm{HEV}$ infection typically is associated with asymptomatic or mild disease, although in immunocompromised individuals genotype $3 \mathrm{HEV}$ causes chronic infection [3]. However, there was a report in Japan [13] of severe hepatitis associated with 8 genotype $3 \mathrm{HEV}$ isolates. Interestingly, three unique mutations (S605P, I978V and V1213A) identified in the ORF1 of these genotype 3 isolates were found to be characteristic of genotype $4 \mathrm{HEV}$ strains, which was considered to be more likely associated with severe hepatitis [13]. Due to the lack of a small animal model for HEV pathogenicity study and a robust cell culture system to study HEV replication, the virus replication fitness or efficiency is often used as a convenient and reliable method to estimate if the mutation(s) in viral genome has any impact on the virus virulence. Therefore, in this study, we aimed to determine if these observed mutations in these genotype $3 \mathrm{HEV}$ isolates affect the replication of HEV in vitro.

By using the HEV GFP replicons system, we first demonstrated that the mutation at amino acid residue 1213 reduced the replication efficiency of both HEV genotype 1 (A1213V) and HEV genotype 3 (V1213A). Our results 

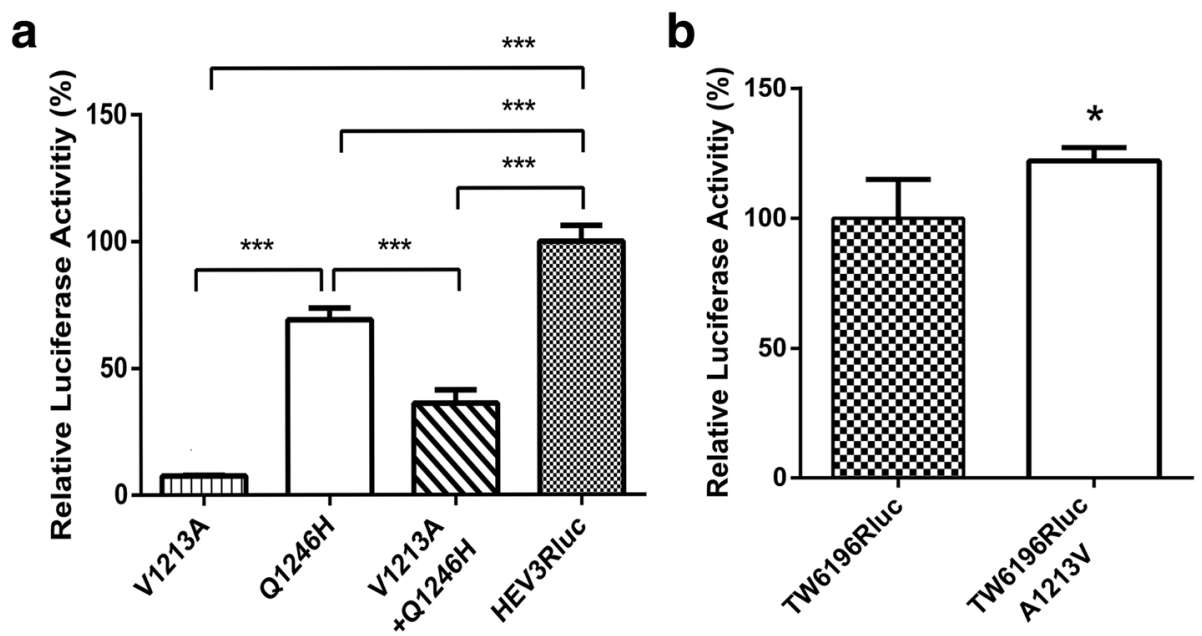

Fig. 4 Relative luciferase activities in Huh7 S10-3 liver cells transfected with (a) RNA of HEV Rluc mutants containing Q1246H and V1213A in HEV genotype 3 Rluc replicons, and (b) RNA of A1213V mutation in genotype 4 Rluc replion. V1213A: mutation of V1213A; Q1246H: mutation of Q1246H; V1213A+ Q1246H: mutation of Q1246H and V1213A; A1213V: mutation of A1213V. are measured at 5 days post-transfection and normalized with cotransfected firefly luciferase RNA. The error bars indicate standard deviations (SD). ${ }^{*}, P<0.05 ;{ }^{* *}, 0.05<P<0.01 ;{ }^{* *}, P<0.001$

indicated that the amino acid residue 1213 is critical for HEV replication, which is consistent with a previous report [13]. However, the replication level of genotype 3 HEV EGFP replicons was low and not very sensitive, as evidenced by the low number of GFP-positive cells and low density of GFP signal, which makes it difficult to more accurately evaluate the replication efficiency in GFP-positive cells. To circumvent the obstacle of the low efficiency of HEV replication, particularly with the genotype 3 HEV EGFP replicon, we subsequently constructed renilla luciferase HEV replicons, which were proved to be more sensitive and accurately reflect the HEV replication level than the HEV EGFP replicons.

The pSHEV3-Rluc genotype 3 HEV replicon system yielded similar, but more reliable, results than that of the HEV EGFP replicons system, demonstrating that the V1213A mutation significantly reduced the replication efficiency of genotype $3 \mathrm{HEV}$, whereas the A1213V mutation slightly decreased the replication efficiency of genotype $1 \mathrm{HEV}$. The amino acid residue 1213 locates in the helicase domain of the HEV ORF1, which has been considered as a critical factor for HEV replication. Since the A1213V mutation in genotype $1 \mathrm{HEV}$ also reduced the viral replication efficiency, suggesting that the V1213 residue is not the optimal residue for genotype $1 \mathrm{HEV}$ replication.

When we examined the effect of other mutations (S605P and 1978V) that were characteristic of genotype 4 HEV on virus replication, we found that S605P and I978V mutations significantly reduced the efficiency of genotype $3 \mathrm{HEV}$ replication $(p<0.001)$. Introduction of the V1213A mutation into the S605P-I978V double mutant further reduced the viral replication efficiency $(p<0.05)$, suggesting that the two sets of mutations have a cumulative, if not synergistic, effect on genotype 3 HEV replication. To further verify that the effect of mutation V1213A is caused by the amino acid, we used a different synonymous codon of the Alanine to create the V1213A mutation in the S605P + I978V mutant, and similar results in HEV replication efficiency were obtained. Taken together, the data suggested, contrary to what we initially thought, that the three mutations identified in the genotype $3 \mathrm{HEV}$ isolates associated with severe hepatitis in Japan actually decreased the efficiency of replication of genotype $3 \mathrm{HEV}$. The S605P and I978V mutations are located in a relatively high variable region of the ORF1 protein, whereas the V1213A mutation is located in the helicase, an enzyme involving in viral replication. Interestingly, here we demonstrated that the Q1246H mutation identified in the high virulent genotype $3 \mathrm{HEV}$ isolates, but the $\mathrm{H}$ residue is different from the corresponding residue typically found in the genotype 4 isolates, only decreased the replication efficiency of genotype $3 \mathrm{HEV}$ at a lower level as compared to that of the V1213A mutation. Our results revealed that the V1213A mutation affects HEV replication efficiency more than that of Q1246H mutation. The V1213A mutation has more drastic effect on HEV replication efficiency, and this may likely due to the effect of the mutation on the function of viral helicase.

Interestingly, when we assessed the effect of A1213V mutation on the replication efficiency of genotype 4 HEV using the genotype $4 \mathrm{HEV}$ luciferase replicon system, we found that the A1213V mutation slightly increased the replication level of genotype $4 \mathrm{HEV}(p<$ $0.05)$. The data suggested that the amino acid residue 
V1213 favors the replication of both zoonotic HEV genotypes 3 and 4, but not genotype $1 \mathrm{HEV}$, as compared to amino acid residue A1213. The V1213A mutation found in the patients infected with the genotype 3 virus isolates may reduce $\mathrm{HEV}$ replication level, and thus was likely not a key factor responsible for the observed severe hepatitis associated with these genotype 3 isolates containing this mutation. HEV infection causes acute viral hepatitis but can also result in chronic hepatitis $\mathrm{E}$ in immunocompromised patients [20]. Genotype $4 \mathrm{HEV}$ was reportedly associated with severe diseases more frequently than genotype $3 \mathrm{HEV}[11,12]$. An insertion of a 58 amino acid residues of human ribosomal protein S17 in the hypervariable region (HVR) of HEV ORF1 apparently enhances HEV replication efficiency in vitro [21]. Together with our data from this study, it appears that both host-specific factors and virus genetic element(s), including virus genotype and mutations, may contribute to HEV pathogenicity.

\section{Conclusion}

The results from this study suggested that the V1213A mutation reduced HEV replication, and is not likely associated with the reported severe hepatitis caused by genotype $3 \mathrm{HEV}$ isolates containing this mutation.

\section{Acknowledgements}

We thank Barbara Dryman for technical assistance, Alicia Feagins for providing the infectious CDNA clone of HEV TW6196, Yao-Wei Huang for providing the pSHEV3 infectious CDNA clone, and Sue Emerson (NIAID, NIH) for providing the infectious CDNA clone of pSK-HEV2 and Huh7 S10-3 liver cells. The authors declare that there is no conflict of interest.

\section{Funding}

This study is supported by grants from the U.S. National Institutes of Health (R01 Al074667 and R01 Al050611). The funding body has no role in the study design, or collection, analysis and interpretation of data, or writing the manuscript.

\section{Availability of data and materials}

Data sharing not applicable to this article as no datasets were generated or analysed during the current study. For request of reagents and materials generated from this study, please contact author.

\section{Authors' contributions}

DC designed the study and conducted all the experiments, and wrote the manuscript. YYN helped perform the mutagenesis and luciferase assay experiments. XJM participated in its design and coordination of the study, and wrote the manuscript. All authors read and approved the final manuscript.

\section{Ethics approval and consent to participate}

Not applicable

\section{Consent for publication}

Not applicable

\section{Competing interests}

The authors declare that they have no competing interests.
Received: 25 May 2017 Accepted: 1 February 2018

Published online: 08 February 2018

\section{References}

1. Cao D, Meng XJ. Molecular biology and replication of hepatitis E virus. Emerg Microbes Infect. 2012;1:e17.

2. Dalton HR, Bendall R, ljaz S, Banks M. Hepatitis E: an emerging infection in developed countries. Lancet Infect Dis. 2008:8:698-709.

3. Kamar N, Abravanel F, Lhomme S, Rostaing L, Izopet J. Hepatitis E virus: chronic infection, extra-hepatic manifestations, and treatment. Clinics and Research in Hepatology and Gastroenterology. 2015;39:20-7.

4. Smith DB, Simmonds P, International Committee on Taxonomy of Viruses Hepeviridae Study G, Jameel S, Emerson SU, Harrison TJ, et al. Consensus proposals for classification of the family Hepeviridae. J Gen Virol. 2014;95: 2223-32.

5. Ohnishi S, Kang JH, Maekubo H, Arakawa T, Karino Y, Toyota J, et al. Comparison of clinical features of acute hepatitis caused by hepatitis $\mathrm{E}$ virus (HEV) genotypes 3 and 4 in Sapporo, Japan. Hepatol Res. 2006;36:301-7.

6. Meng XJ. Hepatitis E virus: animal reservoirs and zoonotic risk. Vet Microbiol. 2010;140:256-65.

7. Meng XJ. From barnyard to food table: the omnipresence of hepatitis E virus and risk for zoonotic infection and food safety. Virus Res. 2011:161:23-30.

8. Rein DB, Stevens GA, Theaker J, Wittenborn JS, Wiersma ST. The global burden of hepatitis E virus genotypes 1 and 2 in 2005. Hepatology. 2012;55: 988-97.

9. van Tong $\mathrm{H}$, Hoan NX, Wang B, Wedemeyer $\mathrm{H}$, Bock $C T$, Velavan TP, Hepatitis E. Virus mutations: functional and clinical relevance. EBioMedicine. 2016;11:31-42.

10. Smith DB, Simmonds P. Hepatitis E virus and fulminant hepatitis-a virus or host-specific pathology? Liver Int. 2015;35:1334-40.

11. Inoue J, Nishizawa T, Takahashi M, Aikawa T, Mizuo H, Suzuki K, et al. Analysis of the full-length genome of genotype 4 hepatitis $E$ virus isolates from patients with fulminant or acute self-limited hepatitis E. J Med Virol. 2006;78:476-84.

12. Jeblaoui A, Haim-Boukobza S, Marchadier E, Mokhtari C, Roque-Afonso AM. Genotype 4 hepatitis e virus in france: an autochthonous infection with a more severe presentation. Clin Infect Dis. 2013:57:e122-6.

13. Takahashi K, Okamoto H, Abe N, Kawakami M, Matsuda H, Mochida S, et al. Virulent strain of hepatitis E virus genotype 3, Japan. Emerg Infect Dis. 2009: 15:704-9.

14. Takahashi M, Nishizawa T, Miyajima H, Gotanda Y, lita T, Tsuda F, et al. Swine hepatitis $\mathrm{E}$ virus strains in Japan form four phylogenetic clusters comparable with those of Japanese isolates of human hepatitis $E$ virus. J Gen Virol. 2003:84:851-62.

15. Huang YW, Opriessnig T, Halbur PG, Meng XJ. Initiation at the third in-frame AUG codon of open reading frame 3 of the hepatitis $E$ virus is essential for viral infectivity in vivo. J Virol. 2007;81:3018-26.

16. Cordoba L, Feagins AR, Opriessnig T, Cossaboom CM, Dryman BA, Huang YW, et al. Rescue of a genotype 4 human hepatitis $E$ virus from cloned CDNA and characterization of intergenotypic chimeric viruses in cultured human liver cells and in pigs. J Gen Virol. 2012;93:2183-94.

17. Pudupakam RS, Huang YW, Opriessnig T, Halbur PG, Pierson FW, Meng XJ. Deletions of the hypervariable region (HVR) in open reading frame 1 of hepatitis $\mathrm{E}$ virus do not abolish virus infectivity: evidence for attenuation of HVR deletion mutants in vivo. J Virol. 2009:83:384-95.

18. Cao D, Huang YW, Meng XJ. The nucleotides on the stem-loop RNA structure in the junction region of the hepatitis $\mathrm{E}$ virus genome are critical for virus replication. J Virol. 2010;84:13040-4.

19. Emerson SU, Nguyen H, Graff J, Stephany DA, Brockington A, Purcell RH. In vitro replication of hepatitis $\mathrm{E}$ virus (HEV) genomes and of an HEV replicon expressing green fluorescent protein. J Virol. 2004:78:4838-46.

20. Meng XJ. Expanding host range and cross-species infection of hepatitis $E$ virus. PLoS Pathog. 2016;12:e1005695

21. Shukla P, Nguyen HT, Faulk K, Mather K, Torian U, Engle RE, et al. Adaptation of a genotype 3 hepatitis $E$ virus to efficient growth in cell culture depends on an inserted human gene segment acquired by recombination. J Virol. 2012;86:5697-707

\section{Publisher's Note}

Springer Nature remains neutral with regard to jurisdictional claims in published maps and institutional affiliations. 